\title{
Concurrent intrathyroidal thymus and parathyroid in a patient with papillary thyroid carcinoma: a challenging diagnosis
}

\author{
Georgios Velimezis', Argyrios loannidis', Sotirios Apostolakis', Maria Chorti², \\ Charalampos Avramidis ${ }^{1}$ and Evripidis Papachristou ${ }^{1}$
}

Departments of 'Surgery and 2Pathology, Sismanoglion Hospital, Amarousion, Greece

Correspondence should be addressed to S Apostolakis

Email

sotapostolakis@gmail.com

\section{Summary}

During embryogenesis, the thymus and inferior parathyroid glands develop from the third pharyngeal pouch and migrate to their definite position. During this process, several anatomic variations may arise, with the thyroid being one of the most common sites of ectopic implantation for both organs. Here, we report the case of a young female patient, who underwent total thyroidectomy for papillary carcinoma of the thyroid. The patient's history was remarkable for disorders of the genitourinary system. Histologic examination revealed the presence of well-differentiated intrathyroidal thymic tissue, containing an inferior parathyroid gland. While each individual entity has been well documented, this is one of the few reports in which concurrent presentation is reported. Given the fact that both the thymus and the inferior parathyroid are derivatives of the same embryonic structure (i.e. the third pharyngeal pouch), it is speculated that the present condition resulted from a failure in separation and migration during organogenesis.

\section{Learning points:}

- Intrathyroidal thymus and parathyroid are commonly found individually, but rarely concurrently.

- It is a benign and asymptomatic condition.

- Differential diagnosis during routine workup with imaging modalities can be challenging.

\section{Background}

The first two months of gestation are considered to be critical for the normal development of the embryo. It is the period in which the four pharyngeal pouches are formed (week 4) and give rise to their derivatives (by the middle of the 8th week). Among the notable organs that arise from these structures of endodermal origin are the thymus and the inferior parathyroid glands, both of which develop from the third pharyngeal pouch and migrate to their definite position. In contrast, the superior parathyroid glands develop from the fourth pharyngeal pouch. On the other hand, the thyroid gland develops around the 24th day of gestation from the primitive pharynx and the neural crest (1).
Several anatomic variations may arise because of discrepancies from normal migration. Hence, it is possible that remnants of the thymus are trapped in the neck during its descend to the superior mediastinum, forming an ectopic focus. Most commonly, ectopic thymus is located at the level of the thyroid gland (2). Intrathyroidal thymus is considered to be a rare entity; however in the work by Fukushima et al. (3), prevalence was found to be about $1 \%$ in Japanese children.

Similarly, the incidence of an ectopic parathyroid gland in a series of 231 patients operated for hyperthyroidism, was as high as $16 \%$ (4), with the inferior parathyroids being more commonly in ectopic 
positions than the superior. The most common location of implantation was the thymus (30\%), followed by the thyroid in $22 \%$ of these cases. Yet, these numbers vary greatly between studies (5).

While both of the aforementioned entities have been studied extensively, there are very few reports of combined malposition of these glands. Hence, in the present paper we report the case of a young female patient with concurrent intrathyroidal thymic tissue, parathyroid gland and papillary adenoma of the thyroid.

\section{Case presentation}

A 26-year-old female patient was admitted to our clinic for an elective thyroidectomy. She had been taking levothyroxine $75 \mu \mathrm{g}$ once daily, and at the time of admission, she was essentially euthyroid. Previous ultrasonography had demonstrated a thyroid gland within normal limits and the presence of an inhomogeneous, $1.15 \mathrm{~cm}$ nodule with micro-calcifications in the right lobe (Fig. 1A), which fine needle aspiration biopsy cytology suggested to be malignant. She subsequently underwent a TC-99M (pertechnetate) thyroid scan with homogenous uptake of the radiopharmaceutical throughout the gland without the presence of any image suggestive of nodule (Fig. 1B). No enlarged lymph nodes or pathology from the surrounding tissues were identified through the computed tomography scan.

Her past history was remarkable for congenital aplasia of the right kidney, polycystic ovarian syndrome and a bicornuate uterus, for which she had had an operation, along with an appendectomy. Upon admission she underwent a thorough laboratory evaluation, with blood tests, electrocardiogram and chest $\mathrm{X}$ ray, which were unremarkable.

\section{Treatment}

The patient underwent total thyroidectomy with no notable incidences during the operation. The postoperative biochemical analysis revealed that $\mathrm{Ca}$ and $\mathrm{P}$ levels were within normal limits.

\section{Outcome and follow-up}

Upon dissection of the specimen, a white focus in the right lobe with a maximum diameter of $0.8 \mathrm{~cm}$ was identified. The histological examination revealed the presence of carcinoma of the thyroid with a papillary pattern with
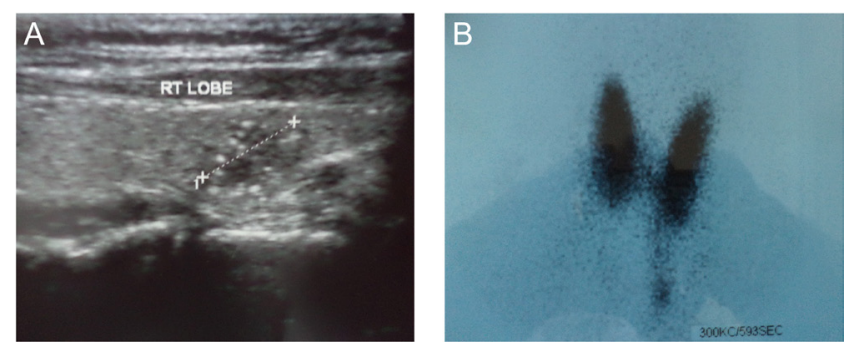

Figure 1

Ultrasonography of the thyroid gland demonstrating an inhomogeneous, $1.15 \mathrm{~cm}$ nodule with micro-calcifications in its right lobe (A) and TC-99M (pertechnetate) thyroid scan with homogenous uptake of the radiopharmaceutical throughout the gland without the presence of any image suggestive of nodule (B).

crowding, overlapping, clearing and grooving nuclei (Fig. 2A) in this mentioned lesion of the right lobe and in multiple smaller sites in both thyroid lobes, compatible with multifocal PTC diagnosis. No infiltration of the capsule was identified. In addition, in the right lobe of the thyroid, an intrathyroidal parathyroid gland (Fig. 2B) was recognized along with a site of intrathyroidal thymic tissue (Fig. 2C and D). The recovery of the patient was uneventful with dismissal on the day following the operation. Two years after the operation the patient remains in good health.
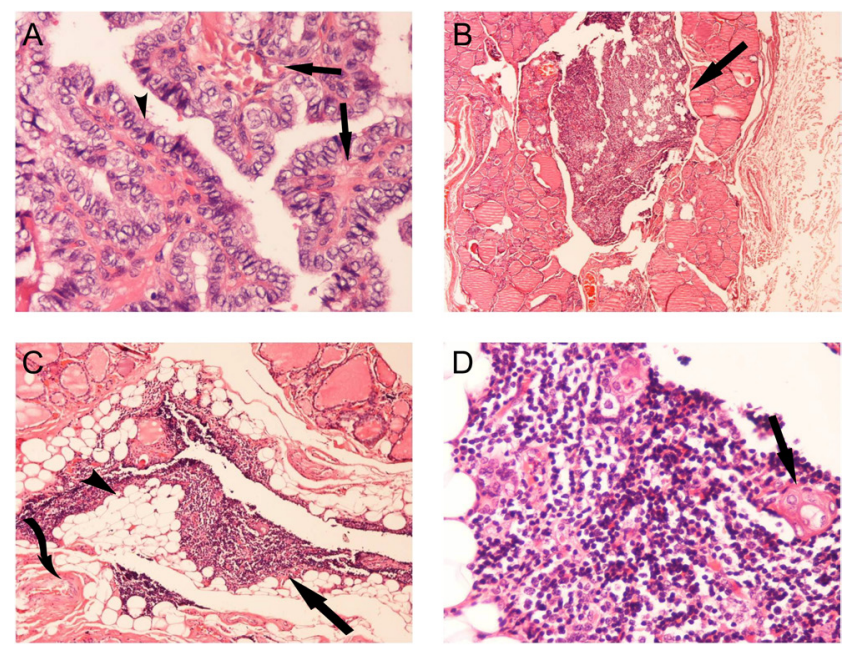

Figure 2

H\&E stained sections from the histological examination of the excised thyroid. Carcinoma of the thyroid with papillary pattern (arrows) and characteristic enlargement, crowding, overlapping, clearing and grooving nuclei (arrowhead) was identified (Image A, mag. $\times 100$ ). Moreover, a parathyroid gland (arrow) was found to be entirely within the thyroid (Image B, mag. ×40). Finally, intrathyroidal/subcapsular (curved arrow) ectopic thymic tissue (arrow) with areas of adipose evolution (arrowhead) (Image C, mag. $\times 100$ ) and Hassall's corpuscle (arrow) was found (Image D, mag. $\times 400$ ). 


\section{Discussion}

In the present paper we report the case of a patient with papillary carcinoma of the thyroid with a coexistent intrathyroidal parathyroid gland and thymic tissue. All lesions were located in the right lobe of the thyroid, with imaging examinations not being able to differentiate between them, as findings consistent with either possible diagnosis were present (6). Eventually, diagnosis was established through histologic examination of the surgically excised tissue.

To the best of our knowledge, such a case with a combination of intrathyroidal parathyroid gland and thymic tissue with coexisting papillary carcinoma of the thyroid has been reported only once before by Kim et al. (7). In their work the authors report the case of a 29 -yearold female patient with a history of right lobectomy due to papillary carcinoma of the thyroid. Her follow-up ultrasonography revealed a hyperechoic nodule, which was later identified by microscopy as ectopic thymic tissue containing a parathyroid gland.

Wu et al. (8) report the microscopic findings from an asymptomatic 22-year-old female patient with a painless cervical mass. Apart from lymphocytic infiltrates, pathological examination revealed the presence of thymic tissue with well-differentiated cortex, medulla and Hassall's corpuscles, inside an intrathyroidal parathyroid gland. Ectopic thymus in both lobes of the thyroid with one site containing a parathyroid gland has also been reported (9). Interestingly, the nodule presented isoechoic in the ultrasound. Finally, in a third patient, an inhomogeneous hypoechoic solitary nodule was found, which from histological examination was identified to be an ectopic focus of thymic tissue surrounding a parathyroid gland (10).

Given the fact that both the thymus and the inferior parathyroid are derivatives of the same embryonic structure (i.e. the third pharyngeal pouch), it is speculated that the present condition resulted from a failure in separation during organogenesis. The majority of information concerning organogenesis from the third pharyngeal pouch comes from experiments in mice. This is because the relevant processes have been shown to be conserved between mouse and human (11). In fact, in the beginning, in each side of the developing pharynx, thymus and inferior parathyroid gland exist as a single primordial structure, which under the effect of specific transcription factors differentiate into two separate organs
$(12,13)$. Then, the thymic component migrates toward the superior mediastinum, while it remains attached to the parathyroid through a cord-like structure. The two thymic primordia (each from either side) then fuse, following their separation from the ipsilateral parathyroid (11).

In our case, since from the histologic examination the two structures were found to be well differentiated, we could speculate that the anomaly occurred during the migration rather than during differentiation of the organs, with part of the unilateral primordial structure being trapped inside the ipsilateral pole of the thyroid. In addition, while no definite genetic association has been established yet between the migration of the third pharyngeal pouch derivatives and the development of the genitourinary system, the possibility of the presence of a syndrome that unifies the aforementioned anomalies is a theory that cannot, yet, be ruled out. But one must have in mind that our patient was born in 1987, too close to Chernobyl nuclear blast on April 26, 1986 that generated a great amount of radioactive iodine 131 linked with thyroid papillary cancer (14). The radioactive exposure is known to produce minisatellite mutations linked with body malformations (15).

In conclusion, intrathyroidal thymic tissue and parathyroid gland are an extremely rare finding, with their recognition being challenging as they do not have consistent clinical presentation or imaging features. Hence, diagnosis can be verified only through histology.

Declaration of interest

The authors declare that there is no conflict of interest that could be perceived as prejudicing the impartiality of the research reported.

\section{Funding}

This research did not receive any specific grant from any funding agency in the public, commercial or not-for-profit sector.

\section{Patient consent}

The authors confirm that written informed consent was obtained from the patient for publication of this article and accompanying images.

\section{Acknowledgements}

The authors would like to thank the patient described for her willingness to participate in this research endeavor. 


\section{References}

1 Policeni BA, Smoker WRK \& Reede DL 2012 Anatomy and embryology of the thyroid and parathyroid glands. Seminars in Ultrasound, CT and MRI 33 104-114. (doi:10.1053/ j.sult.2011.12.005

2 Bale PM \& Sotelo-Avila C 1993 Maldescent of the thymus: 34 necropsy and 10 surgical cases, including 7 thymuses medial to the mandible. Pediatric Pathology 13 181-190. (doi:10.3109/15513819309048205)

3 Fukushima T, Suzuki S, Ohira T, Shimura H, Midorikawa S, Ohtsuru A, Sakai A, Abe M, Yamashita S \& Suzuki S 2015 Prevalence of ectopic intrathyroidal thymus in Japan: the Fukushima health management survey. Thyroid 25 534-537. (doi:10.1089/ thy.2014.0367)

4 Phitayakorn R \& McHenry CR 2006 Incidence and location of ectopic abnormal parathyroid glands. American Journal of Surgery 191 418-423. (doi:10.1016/j.amjsurg.2005.10.049)

5 Noussios G, Anagnostis P \& Natsis K 2012 Ectopic parathyroid glands and their anatomical, clinical and surgical implications. Experimental and Clinical Endocrinology and Diabetes 120 604-610. (doi:10.1055/ s-0032-1327628)

6 Durmaz E, Barsal E, Parlak M, Gurer I, Karaguzel G, Akcurin S \& Bircan I 2012 Intrathyroidal ectopic thymic tissue may mimic thyroid cancer: a case report. Journal of Pediatric Endocrinology and Metabolism 25 997-1000. (doi:10.1515/jpem-2012-0207)

7 Kim A, Kang SH \& Bae YK 2014 Ectopic intrathyroidal thymus accompanied by intrathyroidal parathyroid as a cause of a solitary thyroid nodule in adult. International Journal of Clinical and Experimental Pathology 7 6375-6378.
8 Wu SL, Gupta D \& Connelly J 2001 Adult ectopic thymus adjacent to thyroid and parathyroid. Archives of Pathology and Laboratory Medicine 125 842-843. (doi:10.1043/0003-9985(2001)125<0842:aetatt>2.0.co;2)

9 Lin W-L, Tsai C-H, Liu C-L, Yang L-C \& Chao W-R 2011 Bilateral cervical ectopic thymic nodules with accessory thyroid tissue and an ectopic parathyroid in the neck region. Journal of Dental Sciences $\mathbf{6}$ 61-64. (doi:10.1016/j.jds.2011.02.009)

10 Lignitz S, Musholt TJ, Kreft A, Engel R, Brzezinska R \& Pohlenz J 2008 Intrathyroidal thymic tissue surrounding an intrathyroidal parathyroid gland, the cause of a solitary thyroid nodule in a 6-yearold boy. Thyroid 18 1125-1130. (doi:10.1089/thy.2008.0055)

11 Farley AM, Morris LX, Vroegindeweij E, Depreter ML, Vaidya H, Stenhouse FH, Tomlinson SR, Anderson RA, Cupedo T, Cornelissen JJ et al. 2013 Dynamics of thymus organogenesis and colonization in early human development. Development 140 2015-2026. (doi:10.1242/dev.087320)

12 Gordon J, Bennett AR, Blackburn CC \& Manley NR 2001 Gcm2 and Foxn 1 mark early parathyroid- and thymus-specific domains in the developing third pharyngeal pouch. Mechanisms of Development 103 141-143. (doi:10.1016/S0925-4773(01)00333-1)

13 Zou D, Silvius D, Davenport J, Grifone R, Maire P \& Xua P-X 2006 Patterning of the third pharyngeal pouch into thymus/parathyroid by Six and Eya1. Developmental Biology 293 499-512. (doi:10.1016/j. ydbio.2005.12.015)

14 Bennet P, Repachioli M \& Carr Z 2006 Health effects of the Chernobyl accident and special health care. In Report of the UN Chernobyl Forum Expert Group 'Health'. Geneva: WHO.

15 Dubrova YE, Grant G, Chumak AA, Stezhka VA \& Karakasian AN 2002 Elevated minisatellite mutation rate in the post-chernobyl families from ukraine. American Journal of Human Genetics 71 801-809. (doi:10.1086/342729)

Received in final form 6 May 2017

Accepted 1 June 2017 